\title{
Pitavastatin, an HMG-CoA Reductase Inhibitor, Ameliorates Endothelial Function in Chronic Smokers
}

\author{
Osamu Yoshida, MD; Takahisa Kondo, MD; Yasuko Kureishi-Bando, MD; \\ Tomonori Sugiura, MD*; Kengo Maeda, MD; \\ Kenji Okumura, MD**; Toyoaki Murohara, MD
}

\begin{abstract}
Background: Smoking is a major cardiovascular risk factor, leading to endothelial dysfunction. The present study investigated the hypothesis that pitavastatin, an HMG-CoA reductase inhibitor, may improve endothelial function in chronic smokers via its antioxidant properties.

Methods and Results: The 30 male chronic smokers who exhibited mild hypercholesterolemia at the time of physical check-up were enrolled and randomized to the pitavastatin group $(2 \mathrm{mg} /$ day, $n=15)$ or the untreated control group $(n=15)$. Before and after the 4-week treatment period, endothelium-dependent flow-mediated dilation (FMD) and endothelium-independent dilation by glyceryl trinitrate (GTD) were examined, and the FMD/GTD ratio was calculated. The pitavastatin group showed a significant restoration of endothelial function (percent change in FMD: $+49.6 \%$ vs $+1.4 \%$; percent change in FMD/GTD ratio: $+26.6 \%$ vs $-4.5 \%, P<0.05$ respectively), and a significant reduction in oxidative stress levels (malondialdehyde-low-density lipoprotein-cholesterol: $-16.6 \%$ vs $+7.5 \%$; free radical activity: $-1.8 \%$ vs $+9.7 \%, \mathrm{P}<0.05$ respectively) compared with the control group. Pitavastatin had no effect on the number of circulating $C D 34^{+} \mathrm{CD} 133^{+}$progenitor cells, endothelial progenitor cells, or the MMP-2, MMP-9 and VEGF levels. In vitro oxidative stress monitoring assay revealed that pitavastatin protected endothelial cells against oxidative stress.
\end{abstract}

Conclusions: Pitavastatin restores endothelial function, even in chronic smokers, possibly through its antioxidative properties. (Circ $J$ 2010; 74: 195-202)

Key Words: Cigarette smoking; Endothelial progenitor cells; Oxidative stress; Pitavastatin

$\mathbf{I}$ $\mathrm{t}$ has been established that there is a close link between endothelial dysfunction and accumulation of cardiovascular risk factors, ${ }^{1-3}$ among which smoking is one of the most deleterious and self-preventable. ${ }^{4}$ It has been reported that cigarette smoke contains more than 4,000 substances that augment oxidative stress in vivo. ${ }^{5,6}$ These substances induce denaturation of enzymes, including endothelial nitric oxide synthase (eNOS), leading to reduced NO biosynthesis. ${ }^{6}$ Moreover, the superoxide radicals contained in cigarette smoke extract can directly degrade NO. ${ }^{7}$ Cigarette smoking is also known to inhibit the proliferation of endothelial cells, ${ }^{8}$ so it induces marked endothelial dysfunction and NO degradation, and thus, the coronary vasodilator response to acetylcholine and flow-mediated endothelium-dependent vasodilation of the brachial artery are reduced in chronic smokers. ${ }^{9}$

Another important aspect of cigarette smoking is its effects on circulating progenitor cells (CPCs) or endothelial progenitor cells (EPCs). We previously demonstrated that the numbers of these cells are markedly reduced in chronic smokers, but are rapidly restored by smoking cessation. ${ }^{10}$ The number of circulating EPCs is inversely related to cardiovascular risk accumulation and is positively related to endothelial function. ${ }^{11,12}$ Taken together, smoking seems to impair endothelial function via both oxidative stress and anti-progenitor cell properties.

HMG-CoA reductase inhibitors (so-called statins) have been demonstrated to improve endothelial function. ${ }^{13-15}$

Received May 14, 2009; revised manuscript received July 29, 2009; accepted August 31, 2009; released online November 19 , 2009 Time for primary review: 25 days

Department of Cardiology, Nagoya University Graduate School of Medicine, *Department of Cardio-Renal Medicine and Hypertension, Nagoya City University Graduate School of Medical Science and **Department of Cardiovascular Research Medicine, Nagoya University School of Medicine, Nagoya, Japan

Sources of Funding: A grant from the Smoking Research Foundation to T.M.

Mailing address: Toyoaki Murohara, MD, Department of Cardiology, Nagoya University Graduate School of Medicine, 65 Tsurumai, Showa-ku, Nagoya 466-8550, Japan. E-mail: murohara@med.nagoya-u.ac.jp

ISSN-1346-9843 doi:10.1253/circj.CJ-09-0345

All rights are reserved to the Japanese Circulation Society. For permissions, please e-mail: cj@j-circ.or.jp 
Pitavastatin is a unique lipophilic statin with potent cholesterol-lowering efficacies, and has been shown to improve endothelial function. Specifically, pitavastatin has the beneficial property of being minimally metabolized by the P450 CYP3A4 pathway, which is associated with minimum adverse drug interactions. ${ }^{16}$ In the present study, we investigated its efficacy for restoring the impaired endothelial function in chronic smokers. Moreover, the potential mechanism of pitavastatin-mediated effects on endothelial function were examined, with a special focus into oxidative stress and circulating EPC numbers and functions.

\section{Methods}

\section{Clinical Study}

We enrolled 30 male chronic smokers, who had newly diagnosed mild hypercholesterolemia at the time of physical check-up, after they gave informed consent. Subjects with a history of malignancy, cardiovascular events or active inflammatory diseases, other cardiovascular risk factors or taking other medications were excluded. After enrollment, subjects were randomized to the pitavastatin group or the control untreated group. Subjects in the pitavastatin group received pitavastatin (Kowa Inc, Nagoya, Japan) at a dose of $2 \mathrm{mg}$ once daily for 4 weeks, while the control group was instructed to continue lifestyle management. All subjects had no limits on physical activity. Before and after the 4-week treatment period, each patient underwent measurements of blood pressure and endothelial function, and blood sampling for assessing the number of circulating $\mathrm{CD} 45^{\text {low }} \mathrm{CD} 34^{+} \mathrm{CD} 133^{+}$mononuclear cells (ie, CPCs) and EPCs. The level of physical activity was unchanged in both groups, compared with baseline, as determined from interviews at the end of the study. The study protocol was approved by the Ethics Committee of Nagoya University School of Medicine.

\section{Clinical and Laboratory Data}

Peripheral blood $(10 \mathrm{ml})$ was collected from each subject before and after 4 weeks' treatment. Complete blood cell count, biochemical markers (total cholesterol (TC), lowdensity lipoprotein-cholesterol (LDL-C), high-density lipoprotein-cholesterol, triglycerides, fasting plasma glucose, high-sensitivity C-reactive protein), oxidative stress markers (malondialdehyde-LDL-C (MDA-LDL-C), serum free radical activity) and other markers of vascular responsiveness (vascular endothelial growth factor (VEGF), matrix metalloproteinases 2 and 9) were measured. Smoking status was expressed by the Brinkman index, which was calculated as the number of cigarettes per day multiplied by years of smoking.

\section{Assessment of Endothelial Function}

All subjects were asked to fast for at least $8 \mathrm{~h}$ and to avoid ingesting substances such as caffeine, high-fat foods and vitamin $\mathrm{C}$ on the day of the measurements. Subjects were also asked to avoid smoking and sidestream smoke from cigarettes within $24 \mathrm{~h}$ of the measurements. Assessments of endothelial function were performed at $18.00 \mathrm{~h}$, in a quiet, dark, air-conditioned room (temperature $22-25^{\circ} \mathrm{C}$ ). The subjects lay supine throughout the study. Endothelial function was assessed by measuring the endothelium-dependent flow-mediated dilation (FMD) of the brachial artery by reactive hyperemia and endothelium-independent dilation of the brachial artery induced by glycerol trinitrate (GTD). Both FMD and GTD were measured noninvasively using a highresolution ultrasound apparatus with a $7.5-\mathrm{MHz}$ linear array transducer (Prosound SSD-6500SV; ALOKA Co Ltd, Tokyo, Japan), coupled to computer-assisted analysis software (eTRACKING system, ALOKA), which uses an automated edge detection system for measurement of the brachial artery diameter. The vascular responsiveness of the brachial artery was assessed according to the guidelines of the International Brachial Artery Reactivity Task Force ${ }^{17}$ First, the subject rested and the right arm was fixed in a special arm-holding device. The brachial artery was scanned $5-10 \mathrm{~cm}$ above the elbow and its diameter was automatically tracked. The waveform of diameter changes over the cardiac cycle was displayed in real time using the e-TRACKING system. Brachial artery diameter was measured at baseline, $1 \mathrm{~min}$ after forearm hyperemia (produced by releasing a forearm cuff inflated to $200 \mathrm{mmHg}$ for $5 \mathrm{~min}$ ), and at rest after the subject had been lying quietly for $10 \mathrm{~min}$. The change in vessel diameter was immediately expressed as percent change relative to the baseline vessel diameter before cuff inflation. FMD was automatically calculated as the percent change in peak vessel diameter from the baseline value used for analysis. Next the subject lay quietly for $10 \mathrm{~min}$, allowing the diameter to recover to baseline. After acquiring baseline resting images for $30 \mathrm{~s}$, endothelium-independent dilation of the brachial artery was also quantified at $5 \mathrm{~min}$ after sublingual administration of glycerol trinitrate $(0.15 \mathrm{mg}$; Nihon Kayaku, Tokyo, Japan). The GTD was calculated as the percent change in peak vessel diameter from the baseline value and used for the analysis. Furthermore, to estimate the relative proportion of endothelium-dependent dilation to the maximally achievable dilation because of vascular smooth muscle relaxation, the FMD/GTD ratio was also calculated. Throughout the study, FMD was examined by a single cardiologist (T.S.), who was unaware of the treatment regimen of each subject, using same ultrasound apparatus and probe set. The intra-observer coefficient of variation for the baseline diameter was $2.3 \%$.

\section{Quantification of CPCs and EPCs}

The CPC fraction was quantified by flow cytometry as described previously. ${ }^{10}$ EPCs were isolated and analyzed from each subject by the cell culture method. ${ }^{10}$ Isolated EPCs were characterized as spindle-shaped adherent cells that showed uptake of DiI-acetylated LDL (Molecular Probes, Eugene, OR, USA) and binding of FITC-UEA-1 lectin (Sigma, St Louis, MO, USA). Double-positive adherent cells were visualized by fluorescence microscopy (Biozero BZ8000; Keyence, Osaka, Japan) and the number of these cells was counted in 10 randomly selected microscopic fields.

\section{Pitavastatin Preparation}

Pitavastatin was kindly provided by Kowa Co, Ltd (Nagoya, Japan). It was dissolved in dimethyl sulfoxide (Sigma, Tokyo, Japan) and handled under light-protective conditions. The final concentration of the solvent was less than $1 \%$ (vol/vol\%).

\section{Cell Migration Assay}

Migratory activity of EPCs was assessed using a modified Boyden chamber apparatus (Neuroprobe, Gaithersburg, MD, USA) as described previously. ${ }^{10}$ Briefly, EPCs were harvested at 7 days of culture. Culture medium ( $25 \mathrm{ml})$ supplemented with $1 \%$ fetal bovine serum (FBS) and pitavastatin $(100 \mathrm{nmol} / \mathrm{L})$ and/or recombinant VEGF $(10 \mathrm{ng} / \mathrm{ml}$; R\&D systems, Minneapolis, MN, USA) was placed in the lower chamber of the apparatus. After a polyvinylpyrolidone-free polycarbonate-coated filter was placed upon the lower chamber, EPCs suspended in $50 \mu \mathrm{l}$ of culture medium containing 


\begin{tabular}{|c|c|c|c|}
\hline & $\begin{array}{l}\text { Pitavastatin group } \\
\qquad(n=15)\end{array}$ & $\begin{array}{c}\text { Control group } \\
\qquad(n=15)\end{array}$ & $P$ value \\
\hline Age (years) & 39.7 (3.3) & $38.1(2.2)$ & NS \\
\hline Systolic BP (mmHg) & $119.1(3.6)$ & $120.3(3.1)$ & NS \\
\hline BMI $\left(\mathrm{kg} / \mathrm{m}^{2}\right)$ & $23.6(0.8)$ & $23.0(0.9)$ & NS \\
\hline Brinkman index & $583.3(133.8)$ & $449.3(70.3)$ & NS \\
\hline $\mathrm{TC}(\mathrm{mmol} / \mathrm{L})$ & $5.15(0.22)$ & $4.92(0.25)$ & NS \\
\hline LDL-C (mmol/L) & $3.22(0.21)$ & $3.01(0.25)$ & NS \\
\hline HDL-C (mmol/L) & $1.31(0.08)$ & $1.47(0.08)$ & NS \\
\hline Triglycerides (mmol/L) & $1.71(0.27)$ & $1.33(0.16)$ & NS \\
\hline Fasting blood sugar (mmol/L) & $5.48(0.12)$ & $5.29(0.11)$ & NS \\
\hline MDA-LDL-C (U/L) & $104.3(8.2)$ & $96.5(8.7)$ & NS \\
\hline Serum free radical activity (unit) & $149.9(6.5)$ & $145.3(5.4)$ & NS \\
\hline hs-CRP (mg/L) & $1.81(0.84)$ & $1.02(0.31)$ & NS \\
\hline MMP-2 (ng/ml) & $667.3(26.0)$ & $685.3(22.7)$ & NS \\
\hline MMP-9 (ng/ml) & $43.6(6.3)$ & $43.5(5.4)$ & NS \\
\hline VEGF (pg/ml) & $62.6(11.3)$ & $57.3(7.7)$ & NS \\
\hline
\end{tabular}

Data are mean (SEM).

BP, blood pressure; BMI, body mass index; TC, total cholesterol; LDL-C, low-density lipoprotein-cholesterol; HDL-C, high-density lipoprotein-cholesterol; MDA, malondialdehyde-modified; hs-CRP, high-sensitivity C-reactive protein; MMP, matrix metalloproteinase; VEGF, vascular endothelial growth factor.

\begin{tabular}{|c|c|c|c|}
\hline & $\begin{array}{l}\text { Pitavastatin group } \\
\qquad(n=15)\end{array}$ & $\begin{array}{c}\text { Control group } \\
(n=15)\end{array}$ & $P$ value \\
\hline TC & $-24.4(2.9)$ & $-1.9(2.7)$ & $<0.0001$ \\
\hline LDL-C & $-32.3(3.6)$ & $-3.9(4.1)$ & $<0.0001$ \\
\hline HDL-C & $4.2(3.4)$ & $-1.8(2.5)$ & 0.162 \\
\hline MDA-LDL-C & $-16.6(8.5)$ & $7.5(7.2)$ & 0.04 \\
\hline Serum free radical activity & $-1.8(3.1)$ & $9.7(4.5)$ & 0.043 \\
\hline Triglycerides & $-18.2(10.3)$ & $18.1(16.1)$ & 0.068 \\
\hline hs-CRP & $-17.5(13.9)$ & $289.4(154.1)$ & 0.057 \\
\hline MMP-2 & $3.8(5.5)$ & $-3.8(2.6)$ & 0.228 \\
\hline MMP-9 & $-2.1(9.1)$ & $11.4(12.5)$ & 0.392 \\
\hline VEGF & $38.6(36.9)$ & $47.8(38.4)$ & 0.865 \\
\hline
\end{tabular}

Data are mean (SEM).

Abbreviations see in Table 1.

$1 \%$ FBS and pitavastatin were placed in the upper chamber, and incubated for $4 \mathrm{~h}$ at $37^{\circ} \mathrm{C}$ in a humidified incubator. After removal of non-migrated EPCs, the filters were fixed with methanol and stained with May-Gruenwald's solution (Merck, Darmstadt, Germany) and Giemsa stain solution (Sigma). The number of migrating EPCs was then counted in 5 randomly selected microscopic fields. All experiments were performed in triplicate.

\section{Endothelial Network Formation in Basement Matrix Gel}

At day 7 of culture, EPCs $\left(6 \times 10^{3}\right.$ cells/well) were labeled with a green fluorescent dye PKH2-GL (Sigma) and cocultured with unlabeled human umbilical vein endothelial cells (HUVECs: $6 \times 10^{4}$ cells/well) on growth-factor-reduced Matrigel (BectonDickinson, Tokyo, Japan). Cocultured EPCs and HUVECs were incubated at $37^{\circ} \mathrm{C}$ for $12 \mathrm{~h}$. The number of labeled EPCs incorporated into the HUVEC tube formation was assessed in 5 random high-power fields $(\times 100)$ per group.

\section{Estimation of Serum Oxidative Stress}

Serum oxidative free radical levels were estimated using a modified method reported by Alberti et al. ${ }^{18}$ In brief, serum or hydrogen peroxide $(5 \mu \mathrm{l})$ were dissolved in $140 \mu \mathrm{l}$ of acetate buffer $(\mathrm{pH} 4.8)$ in the wells of a 96-well plate and incubated at $37^{\circ} \mathrm{C}$ for $5 \mathrm{~min}$. These solutions were then added to the $100 \mu \mathrm{l}$ of the resulting solutions containing $3.8 \mathrm{mg} / \mathrm{ml}$ of N,N,diethyl-para-phenylendiamine and $4.2 \mathrm{mmol} / \mathrm{L}$ of $\mathrm{FeCl} 2$, and incubated at $37^{\circ} \mathrm{C}$ for $1 \mathrm{~min}$. The absorbance at $505 \mathrm{~nm}$ of these solutions was monitored for $180 \mathrm{~s}$. The serum free racial activity was estimated by comparing the degree of increase in absorbance by the serum to that of increase by hydrogen peroxide. Arbitrarily, 1 unit of total reactive oxygen species (ROS) in the sample was defined to have the same oxidative activity of $1.0 \mathrm{mg} \mathrm{H}_{2} \mathrm{O}_{2} / \mathrm{L}$. Serum MDA-LDL-C by ELISA was measured as another marker of the oxidation level in patients, according to the manufacturer's instructions (Daiichi, Tokyo, Japan).

\section{Detection of Oxidative Stress in HUVECs}

The in-vitro oxidative stress level was detected by an intracellular ROS assay system using fluorescent dye 5-6-chloromethyl-2',7'-dichlorodihydrofluorescein diacetate (H2DCFDA; 


\begin{tabular}{|c|c|c|c|}
\hline & $\begin{array}{l}\text { Pitavastatin group } \\
\qquad(n=15)\end{array}$ & $\begin{array}{c}\text { Control group } \\
(n=15)\end{array}$ & $P$ value \\
\hline Diameter before RH $(\mathrm{mm})$ & $4.5 \pm 0.2$ & $4.4 \pm 0.1$ & 0.683 \\
\hline Diameter after RH (mm) & $4.6 \pm 0.2$ & $4.6 \pm 0.1$ & 0.804 \\
\hline FMD (\%) & $3.6 \pm 0.4$ & $4.5 \pm 0.5$ & 0.211 \\
\hline Diameter before GTN $(\mathrm{mm})$ & $4.4 \pm 0.2$ & $4.5 \pm 0.1$ & 0.874 \\
\hline Diameter after GTN (mm) & $5.1 \pm 0.2$ & $5.1 \pm 0.1$ & 0.873 \\
\hline GTD (\%) & $14.2 \pm 1.2$ & $13.8 \pm 1.4$ & 0.845 \\
\hline
\end{tabular}

Data are mean \pm SEM.

$\mathrm{RH}$, reactive hyperemia; FMD, endothelium-dependent flow-mediated dilation; GTN, glycerol trinitrate; GTD, endothelium-independent dilation of the brachial artery induced by glycerol trinitrate.

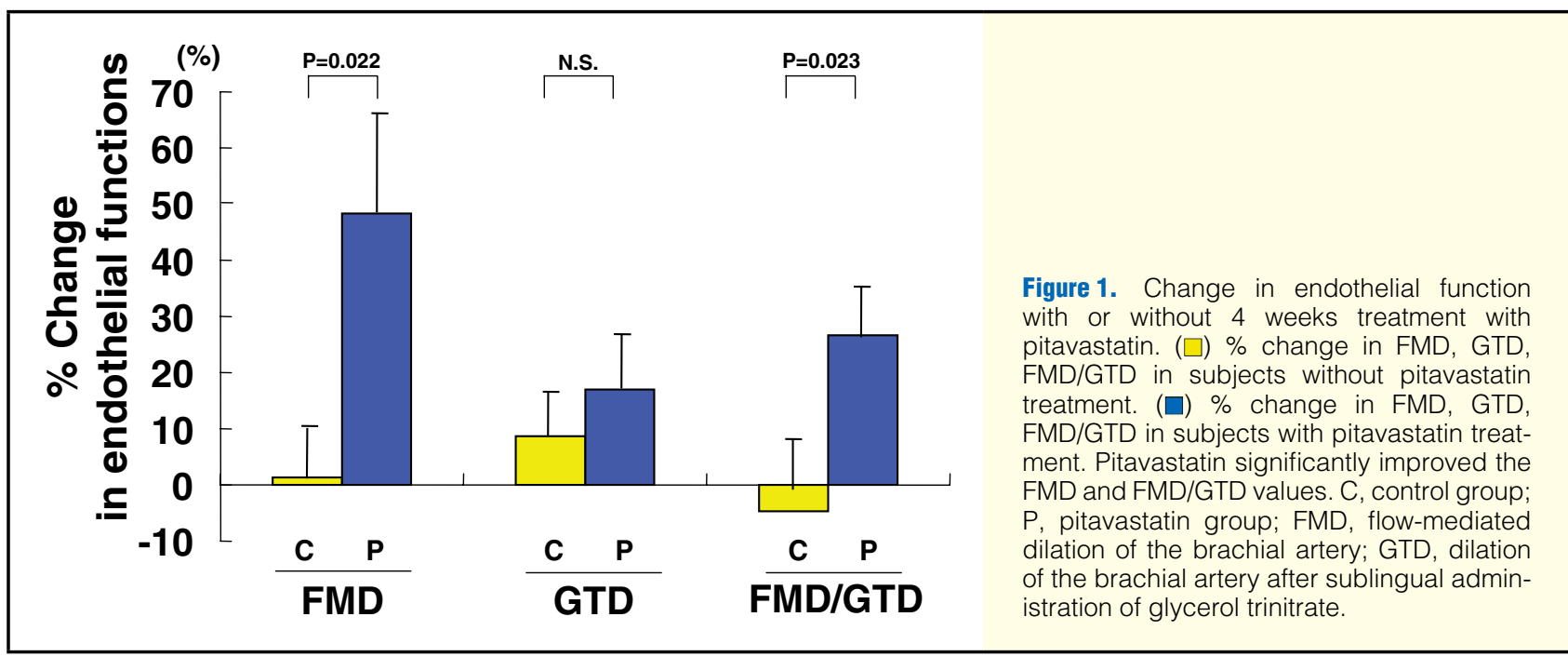

Molecular Probes). HUVECs were loaded with $2 \mu \mathrm{mol} / \mathrm{L}$ of the dye at $37^{\circ} \mathrm{C}$ in humidified conditions for $15 \mathrm{~min}$, followed by incubation with Hoechst 33342 for $5 \mathrm{~min}$. Changes in fluorescence were examined by fluorescence microscopy and a fluorescent plate reader (Fluoroskan Ascent FL; Labsystems, Helsinki, Finland) with excitation and emission at wavelengths of $485 \mathrm{~nm}$ and $527 \mathrm{~nm}$, respectively.

\section{Statistical Analysis}

Results are expressed as mean \pm SEM. Comparison of continuous variables in the clinical study was performed by Student's t-test or the paired t-test as appropriate. Correlations between the \% change of FMD or GTD or the FMD/GTD ratio, and biochemical parameters, were assessed by calculating Pearson's correlation coefficient (r). Furthermore, to identify factors that account for changes in endothelial function, forward stepwise multiple regression analysis was performed using age, systolic blood pressure, body mass index, presence of diabetes mellitus, serum LDL-C and MDA-LDL-C levels and serum free radical activity as covariates. In all analyses, $\mathrm{P}<0.05$ (2-sided) was considered statistically significant.

\section{Results}

\section{Baseline Characteristics of Study Subjects}

There were no significant differences between the 2 groups in any clinical parameters (Table $\mathbf{1}$ ).

\section{Serum Cholesterol, Inflammatory and Oxidative Stress Marker Levels}

As shown in Table 2, in the pitavastatin group, the percent reductions in TC and LDL-C levels and oxidative stress markers, such as the MDA-LDL-C level and serum free radical activity, were significantly larger compared with the control group (TC: $-24.4 \pm 2.9 \%$ vs $-1.9 \pm 2.7 \%$; LDL-C: $-32.3 \pm 3.6 \%$ vs $-3.9 \pm 4.1 \%$; MDA-LDL-C: $-16.6 \pm 8.5 \%$ vs $+7.5 \pm 7.2 \%$; and serum free radical activity: $-1.8 \pm 3.1 \%$ vs $+9.7 \pm 4.5 \%$ in the pitavastatin vs the control group).

\section{Effects of Pitavastatin on Endothelial Function}

At baseline, systolic blood pressure, vessel diameter before and after FMD measurement, vessel diameter before and after GTD measurement, and the FMD and GTD values were not different between the 2 groups (Table 3 ). The effects of pitavastatin on FMD, GTD and the FMD/GTD ratio were evaluated. Percent changes in FMD and the FMD/GTD ratio were significantly larger in the pitavastatin group compared with the control group $(49.6 \pm 17.6 \%$ vs $1.4 \pm 9.1 \%, 26.6 \pm 9.2 \%$ vs $-4.5 \pm 9.0 \% ; \mathrm{P}=0.022, \mathrm{P}=0.023$, respectively) (Figure 1). However, percent changes in GTD was not significantly different between the 2 groups $(17.4 \pm 9.5 \%$ vs $10.5 \pm 7.9 \%$; $\mathrm{P}=0.581)$ Single regression analysis revealed that the percent changes in the LDL-C and MDA-LDL-C levels significantly correlated with the percent changes in the FMD/GTD ratio. To identify the factors by which pitavastatin improved the FMD/GTD value, multiple linear regression analyses with a forward stepwise selection procedure were performed. The 


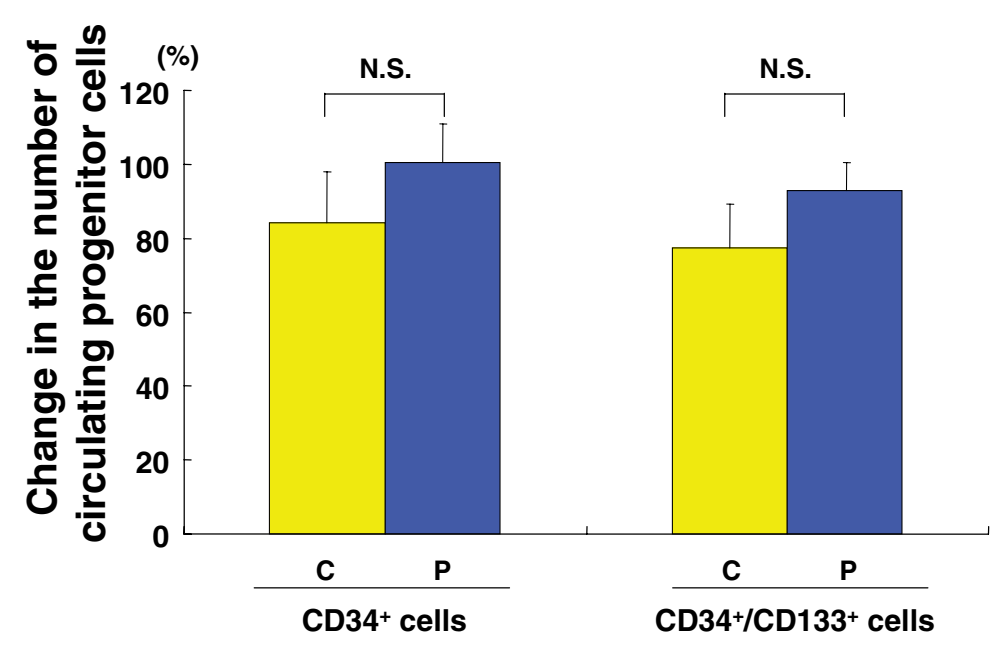

Figure 2. Changes in the number of CD34+ cells and CD34+CD133+ cells in the control (C) and pitavastatin (P) groups. There were no significant differences between the groups in the change in the number of circulating progenitor cells.

A

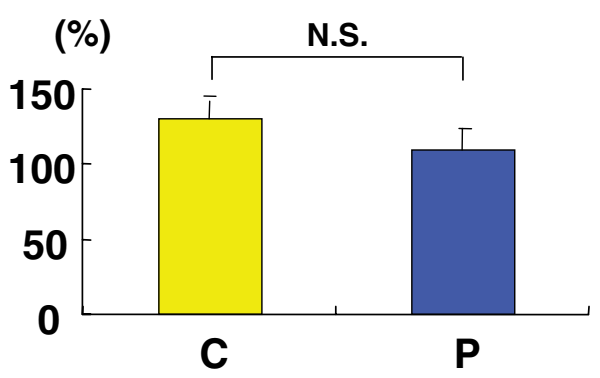

C

Incorporated EPCs

(\%)

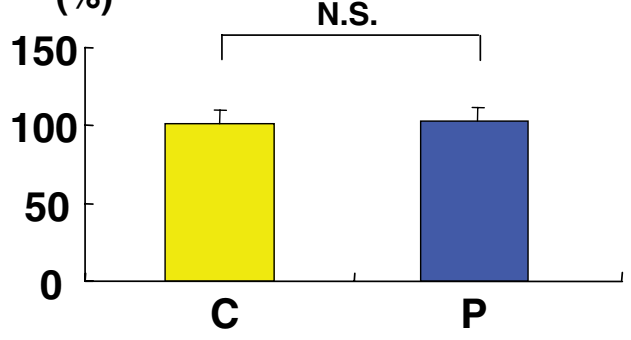

B

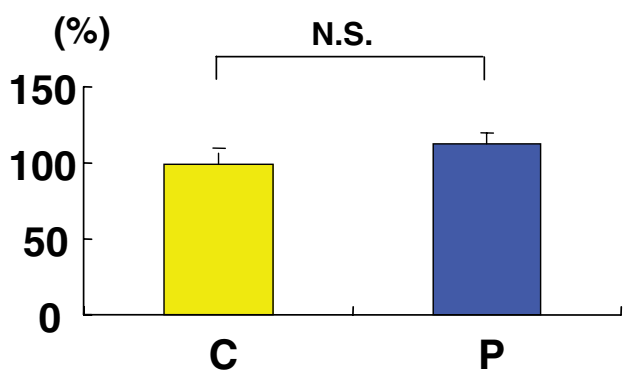

Figure 3. Change in the number and functions of endothelial progenitor cells (EPCs) between the control (C) and pitavastatin (P) groups. (A) Number of EPCs (double positive for acetylated-low density lipoprotein uptake and Dil-lectin labeling) did not differ between groups. (B) EPC migration activity as assessed by modified Boyden chamber did not differ between groups. (C) Number of EPCs incorporated into tube-like networks by HUVECs in Matrigel was not different between the group. HUVECs, human umbilical vein endothelial cells.

findings indicate that the percent changes in LDL-C level, systolic blood pressure, MDA-LDL-C level and serum free radical activity were independently associated with the improvement in the percent change in the FMD/GTD ratio.

\section{Effects of Pitavastatin on the Number and Function of CPCs/EPCs}

At baseline, the number of CPCs and EPCs did not differ between the 2 groups (CPCs: $173.0 \pm 31.7 / 10^{6}$ cells vs 198.6 $\pm 35.8 / 10^{6}$ cells; EPCs: 718.8 $\pm 94.6 / 10$ fields vs $629.8 \pm 58.5 / 10$ fields in the pitavastatin vs the control group). Percent changes in the number of CPCs and EPCs were not different between the pitavastatin and control groups $(-7.2 \pm 7.7 \%$ vs $-22.6 \pm 11.8 \%, 9.1 \pm 14.4 \%$ vs $30.1 \pm 12.1 \%$; $\mathrm{P}=0.29, \mathrm{P}=0.28$, respectively), suggesting pitavastatin had no effect on CPC mobilization (Figure 2). The angiogenic 


\section{A}

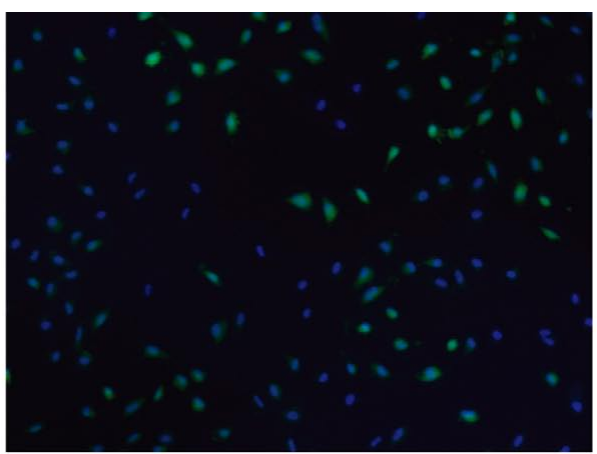

C

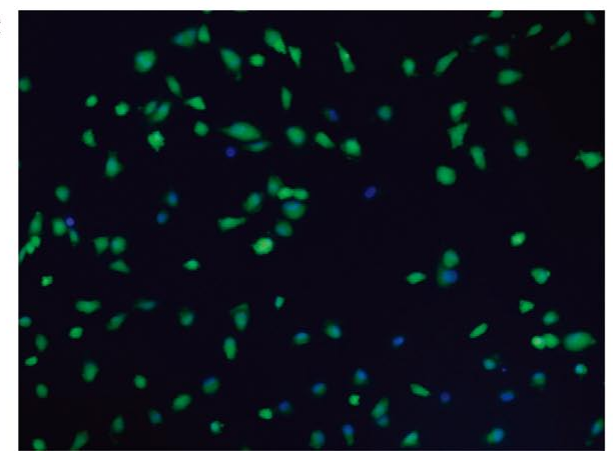

B

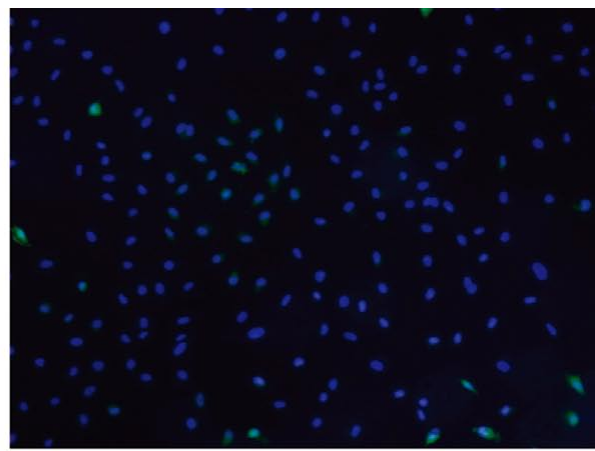

D

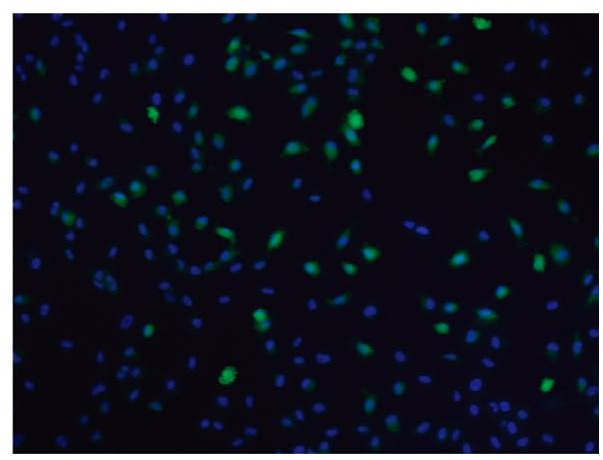

$\mathbf{E}$

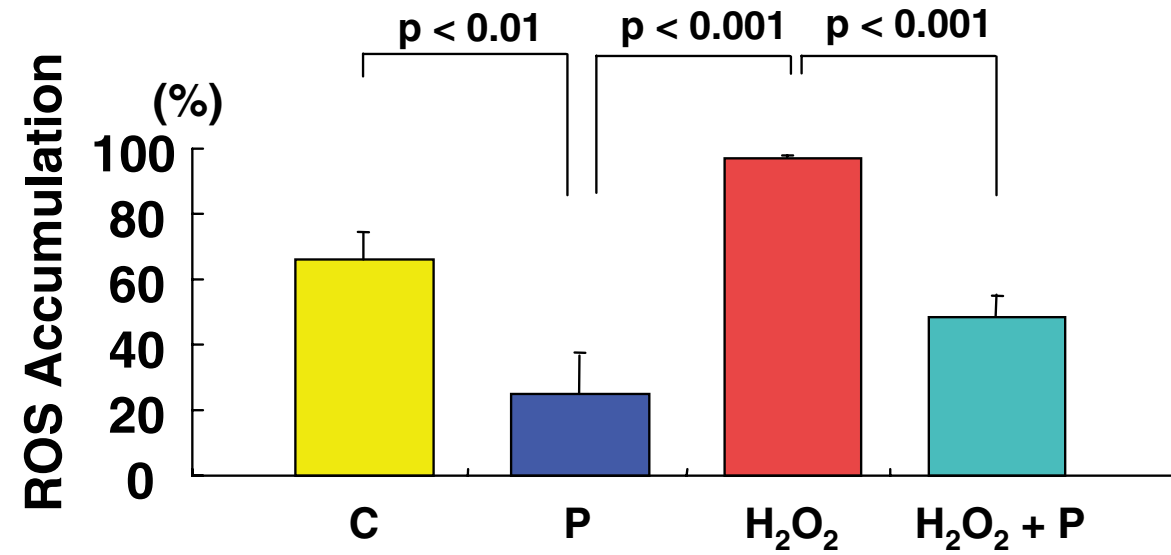

Figure 4. Effects of pitavastatin on oxidative stress production in human umbilical vein endothelium cells (HUVECs). Representative images of intracellular reactive oxygen species (ROS) accumulated in HUVECs. Oxidative stress is indicated as positive green fluorescent dye. Nucleus is stained with DAPI. (A) At baseline, there are few ROS-positive HUVECs. (B) ROS production in HUVECs treated with pitavastatin is significantly reduced compared with the control HUVECs. (C) ROS production in HUVECs is prominent at $15 \mathrm{~min}$ after exposure to $\mathrm{H}_{2} \mathrm{O}_{2}(500 \mu \mathrm{mol} / \mathrm{L})$. (D) Pitavastatin $(100 \mathrm{nmol} / \mathrm{L})$ prevented ROS accumulation in HUVECs at $15 \mathrm{~min}$ after exposure to $\mathrm{H}_{2} \mathrm{O}_{2}(500 \mu \mathrm{mol} / \mathrm{L})$. (E) Summary of data. Data are shown as the mean \pm SEM of triplicate experiments. C, control group; P, pitavastatin group.

functions of isolated EPCs, measured by migration assay and network formation assay using coculture with HUVECs, did not differ significantly between the pitavastatin and control groups (Figure 3).

\section{Effects of Pitavastatin on ROS Accumulation and Viability of HUVECS}

Previous studies indicated that statins have antioxidant properties, ${ }^{19,20}$ so we hypothesized that pitavastatin might improve endothelial function through suppression of oxidative stress in the endothelium of chronic smokers. Intracellular accumu- lation of ROS was monitored using a fluorescent dye indicator, H2DCF-DA. Pitavastatin significantly blocked intracellular oxidative stress accumulation, even without $\mathrm{H}_{2} \mathrm{O}_{2}$ stimulation. After exposure to $500 \mu \mathrm{mol} / \mathrm{L} \mathrm{H}_{2} \mathrm{O}_{2}$, HUVECs showed a significant increase in oxidative stress and these increases in ROS accumulation in the presence of exogenous $\mathrm{H}_{2} \mathrm{O}_{2}$ were significantly inhibited by cotreatment with $100 \mathrm{nmol} / \mathrm{L}$ pitavastatin (Figure 4). Taken together, our results suggest that pitavastatin likely protected the endothelium against oxidative stress, leading to restoration of endothelial function. 


\section{Discussion}

The major findings of the present study are as follows. (1) In chronic smokers, endothelial function as assessed by FMD was impaired and treatment with pitavastatin for 4 weeks significantly improved it. (2) Pitavastatin treatment significantly decreased the serum MDA-LDL-C level, a marker of LDL oxidization, and serum free radical activity. (3) The number and angiogenic functions of $\mathrm{CPC} / \mathrm{EPC}$ in chronic smokers did not change after treatment with pitavastatin. (4) Pitavastatin directly protected endothelial cells against oxidative stress in vitro.

Smoking is known to induce endothelial dysfunction, leading to the development of arteriosclerosis and cardiovascular events. ${ }^{4}$ To date, several mechanisms relating smoking and endothelial dysfunction have been presented. Among these, oxidative stress is considered a primary cause of the endothelial dysfunction induced by smoking; $;, 21$ for example, we previously demonstrated that cigarette smoke extract induced vasoconstriction of isolated porcine coronary arteries by superoxide-mediated degradation of endotheliumderived NO. ${ }^{7}$

Meantime, HMG-CoA reductase inhibitors (statins) have been shown to counteract the harmful influence of smoking through their pleiotropic effects, such as antioxidative and antiinflammatory properties. ${ }^{19,22}$ Atorvastatin has been shown to restore endothelial function in chronic smokers; ${ }^{23}$ however, it as well as other lipophilic statins are metabolized by cytochrome P-450 (CYP) 3A4, and they have been reported to increase the risk of statin-associated adverse reactions when coadministered with other drugs that share CYP3A4 metabolism. ${ }^{24}$ In contrast, pitavastatin is not metabolized by $\mathrm{P}-450$ CYP3A4 and can avoid potential harmful drug interactions. ${ }^{16}$ Our present study clearly demonstrates that pitavastatin treatment for 4 weeks significantly improved endothelial function in chronic smokers, suggesting a possible role in reducing future cardiovascular events in chronic smokers not only by lowering LDL-C levels but also by improving endothelial function.

There are potentially 2 mechanisms by which pitavastatin improves endothelial function: anti-oxidative properties or CPC/EPC-promoting efficacies. Therefore, we examined each in the present study. First, it is well known that statins elicit antioxidative properties; for example, pitavastatin has been shown to attenuate diabetes-induced oxidative stress in vitro and in vivo by inhibiting the activity of Rac- 1,25 a key regulatory molecule for NADPH oxidase. Pitavastatin can also raise the vascular content of tetrahydrobiopterin, ${ }^{26}$ an important allosteric cofactor for $e N O S$. In the present study, MDA-LDL-C levels and serum free radical activity, representative markers of serum oxidative stress, were significantly suppressed by treatment with pitavastatin. Pitavastatin also blocked intracellular oxidative stress in HUVECs at baseline, as well as under ROS-augmented conditions using exogenous $\mathrm{H}_{2} \mathrm{O}_{2}$, which mimic endothelium damaged by excessive oxidative stress in chronic smokers. These findings suggest that pitavastatin improves endothelial function via its antioxidative properties.

Second, statins can promote EPC mobilization from the bone marrow and ameliorate EPCs' function, leading to vascular protection. ${ }^{27,28}$ However, neither the number nor the angiogenic functions of EPCs differed between the pitavastatin and control groups in the present study. The present findings indicate that pitavastatin had almost no effect on the circulating number and functions of EPC in chronic smokers.
Other statins, such as atorvastatin or simvastatin, have been shown to stimulate mobilization of EPCs. The precise reason for the discrepancy is unknown, but there are several possible reasons. Firstly, our study patients were all chronic smokers, and we previously showed that circulating EPC numbers are severely reduced in chronic smokers. ${ }^{10}$ Thus, it is probable that short-term treatment with pitavastatin was not long enough to restore the circulating EPC count. Secondly, the number of study patients was small and it may be necessary to study more number of subjects to see the effects of pitavastatin on progenitor cells.

In conclusion, pitavastatin likely improves endothelial function in chronic smokers by reducing intracellular oxidative stress through inhibition of vascular NADPH oxidase activity and augmentation of $e N O S$ activity, although the possible contribution of improved EPC kinetics and functions could not be confirmed in the present study. Our results suggest a potential role for pitavastatin therapy in the restoration of endothelial function and reduction of future cardiovascular events in high-risk subjects such as chronic smokers. However, we do not want to give any suggestion that pitavastatin intake can overcome the harmful effects of smoking on endothelial function and the most important message is still "smoking cessation".

\section{Acknowledgments}

We thank Mika Aoki and Rie Miura for their technical assistance.

\section{References}

1. Suwaidi JA, Hamasaki S, Higano ST, Nishimura RA, Holmes DR $\mathrm{Jr}$, Lerman A. Long-term follow-up of patients with mild coronary artery disease and endothelial dysfunction. Circulation 2000; 101: 948-954.

2. Schachinger V, Britten MB, Zeiher AM. Prognostic impact of coronary vasodilator dysfunction on adverse long-term outcome of coronary heart disease. Circulation 2000; 101: 1899-1906.

3. Perticone F, Ceravolo R, Pujia A, Ventura G, Iacopino S, Scozzafava A, et al. Prognostic significance of endothelial dysfunction in hypertensive patients. Circulation 2001; 104: 191-196.

4. Kannel WB. Curbing the tobacco menace. Circulation 1997; 96: 1070.

5. Cross CE, O'Neill CA, Reznick AZ, Hu ML, Marcocci L, Packer $\mathrm{L}$, et al. Cigarette smoke oxidation of human plasma constituents. Ann NY Acad Sci 1993; 686: 72-89.

6. Barua RS, Ambrose JA, Srivastava S, DeVoe MC, Eales-Reynolds LJ. Reactive oxygen species are involved in smoking-induced dysfunction of nitric oxide biosynthesis and upregulation of endothelial nitric oxide synthase: An in vitro demonstration in human coronary artery endothelial cells. Circulation 2003; 107: $2342-$ 2347.

7. Murohara T, Kugiyama K, Ohgushi M, Sugiyama S, Yasue H. Cigarette smoke extract contracts isolated porcine coronary arteries by superoxide anion-mediated degradation of EDRF. Am J Physiol 1994; 266: H874-H880.

8. Ockene IS, Miller NH. Cigarette smoking, cardiovascular disease, and stroke: A statement for healthcare professionals from the American Heart Association. American Heart Association Task Force on Risk Reduction. Circulation 1997; 96: 3243-3247.

9. Zeiher AM, Schachinger V, Minners J. Long-term cigarette smoking impairs endothelium-dependent coronary arterial vasodilator function. Circulation 1995; 92: 1094-1100.

10. Kondo T, Hayashi M, Takeshita K, Numaguchi Y, Kobayashi K, Iino $\mathrm{S}$, et al. Smoking cessation rapidly increases circulating progenitor cells in peripheral blood in chronic smokers. Arterioscler Thromb Vasc Biol 2004; 24: 1442 - 1447.

11. Hill JM, Zalos G, Halcox JP, Schenke WH, Waclawiw MA, Quyyumi AA, et al. Circulating endothelial progenitor cells, vascular function, and cardiovascular risk. N Engl J Med 2003; 348: $593-600$.

12. Kajiguchi M, Kondo T, Izawa $\mathrm{H}$, Kobayashi M, Yamamoto K, Shintani S, et al. Safety and efficacy of autologous progenitor cell transplantation for therapeutic angiogenesis in patients with critical 
limb ischemia. Circ J 2007; 71: 196-201.

13. O'Driscoll G, Green D, Taylor RR. Simvastatin, an HMG-coenzyme A reductase inhibitor, improves endothelial function within 1 month. Circulation 1997; 95: 1126-1131.

14. Wassmann S, Faul A, Hennen B, Scheller B, Bohm M, Nickenig G. Rapid effect of 3-hydroxy-3-methylglutaryl coenzyme a reductase inhibition on coronary endothelial function. Circ Res 2003; 93: e98-e103.

15. Takashima H, Ozaki Y, Yasukawa T, Waseda K, Asai K, Wakita $\mathrm{Y}$, et al. Impact of lipid-lowering therapy with pitavastatin, a new HMG-CoA reductase inhibitor, on regression of coronary atherosclerotic plaque: A 3-Dimensional intravascular ultrasound study. Circ J 2007; 71: 1678-1684.

16. Hayashi T, Rani PJ, Fukatsu A, Matsui-Hirai H, Osawa M, Miyazaki A, et al. A new HMG-CoA reductase inhibitor, pitavastatin remarkably retards the progression of high cholesterol induced atherosclerosis in rabbits. Atherosclerosis 2004; 176: 255-263.

17. Corretti MC, Anderson TJ, Benjamin EJ, Celermajer D, Charbonneau F, Creager MA, et al. Guidelines for the ultrasound assessment of endothelial-dependent flow-mediated vasodilation of the brachial artery: A report of the International Brachial Artery Reactivity Task Force. J Am Coll Cardiol 2002; 39: 257-265.

18. Alberti A, Bolognini L, Macciantelli D, Caratelli M. The radical cation of N,N-diethyl-para-phenylendiamine: A possible indicator of oxidative stress in biological samples. Res Chem Intermed 2000; 26: $253-267$.

19. Shishehbor MH, Brennan ML, Aviles RJ, Fu X, Penn MS, Sprecher DL, et al. Statins promote potent systemic antioxidant effects through specific inflammatory pathways. Circulation 2003; 108: $426-431$.

20. Schupp N, Schmid U, Heidland A, Stopper H. Rosuvastatin protects against oxidative stress and DNA damage in vitro via upregulation of glutathione synthesis. Atherosclerosis 2008; 199: 278-287.
21. Tanriverdi H, Evrengul H, Kuru O, Tanriverdi S, Seleci D, Enli Y, et al. Cigarette smoking induced oxidative stress may impair endothelial function and coronary blood flow in angiographically normal coronary arteries. Circ J 2006; 70: $593-599$.

22. Yamada T, Node K, Mine T, Morita T, Kioka H, Tsukamoto Y, et al. Long-term effect of atorvastatin on neurohumoral activation and cardiac function in patients with chronic heart failure: A prospective randomized controlled study. Am Heart J 2007; 153: 1055.e1-e8.

23. Beckman JA, Liao JK, Hurley S, Garrett LA, Chui D, Mitra D, et al. Atorvastatin restores endothelial function in normocholesterolemic smokers independent of changes in low-density lipoprotein. Circ Res 2004; 95: 217-223.

24. Thompson PD, Clarkson P, Karas RH. Statin-associated myopathy. JAMA 2003; 289: $1681-1690$.

25. Tsubouchi H, Inoguchi T, Sonta T, Sato N, Sekiguchi N, Kobayashi $\mathrm{K}$, et al. Statin attenuates high glucose-induced and diabetes-induced oxidative stress in vitro and in vivo evaluated by electron spin resonance measurement. Free Radic Biol Med 2005; 39: 444-452.

26. Shinozaki K, Nishio Y, Ayajiki K, Yoshida Y, Masada M, Kashiwagi A, et al. Pitavastatin restores vascular dysfunction in insulin-resistant state by inhibiting NAD $(\mathrm{P}) \mathrm{H}$ oxidase activity and uncoupled endothelial nitric oxide synthase-dependent superoxide production. J Cardiovasc Pharmacol 2007; 49: 122-130.

27. Llevadot J, Murasawa S, Kureishi Y, Uchida S, Masuda H, Kawamoto A, et al. HMG-CoA reductase inhibitor mobilizes bone marrow-derived endothelial progenitor cells. J Clin Invest 2001; 108: $399-405$

28. Shao H, Tan Y, Eton D, Yang Z, Uberti MG, Li S, et al. Statin and stromal cell-derived factor-1 additively promote angiogenesis by enhancement of progenitor cells incorporation into new vessels. Stem Cells 2008; 26: 1376-1384. 